\title{
Highlights in CAR T-Cell Therapy from EHA2021: Paving the Way for Better Treatments
}

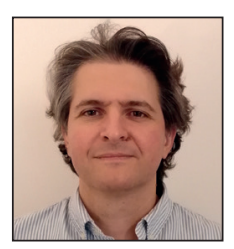

Dr Federico Simonetta

Division of Hematology

University Hospital of Geneva (HUG)

Geneva, Switzerland

\section{CAR T-cell therapy in DLBCL}

Before the approval of the first chimeric antigen receptor (CAR) T-cell therapy for patients with diffuse large B-cell lymphoma (DLBCL) in Europe, treatment options after the first and second line were limited. Thanks to the approval of CAR T-cell therapies, there is a new set of options to treat patients with DLBCL. Currently, there are three CD19-targeted CAR T therapies approved in the United States for the treatment of patients with relapsed or refractory (R/R) DLBCL, namely tisagenlecleucel $(\text { tisa-cel) })^{1}$, axicabtagene ciloleucel (axi-cel) $)^{2}$, and lisocabtagene maraleucel (liso-cel) $)^{3}$. Only tisa-cel ${ }^{4}$ and axi-cel ${ }^{5}$ are authorized so far for the treatment of this patient population in Switzerland. The approvals were based on the results of three pivotal trials, namely, JULIET ${ }^{6}, Z^{2} U M A-1^{7}$, and TRANSCEND-001 ${ }^{8}$, which assessed the efficacy and safety of tisa-cel, axi-cel and liso-cel, respectively, in heavily pretreated patients with R/R DLBCL. Interestingly, the estimated progression-free survival (PFS) rate was approximately $40 \%$ in all three studies. However, further real-life studies are needed to investigate the effect of these CAR T-cell therapies in this patient population.

At EHA2021, the first results of DLBCL patients treated with CAR T-cells from the DESCAR-T registry, the French real-life database were presented. ${ }^{9}$ In this multicenter, retrospective analysis, a total of 550 patients were treated with either tisa-cel $(n=200)$ or axi-cel $(n=350)$. So far, this is the largest reported data series in this patient population; however, with a limited median follow-up of 6.5 months, the long-term effect of the CAR T-cell treatment cannot be evaluated. At 6 months, the PFS rate $(44.5 \%)$ was comparable with the previously reported results from the pivotal studies with tisa-cel ${ }^{6}$ and axi-cel $^{7}$. Due to the large size of the cohort, patients were stratified based on the remission status, a requirement of bridging therapy and progressive disease (PD) at the time of CAR T-cell injection. Of note, PD was clearly indicated as a predictor of poor outcome. Among patients who had PD at the time of treatment, a $38.2 \%$
DOI: 10.36000/HBT.OH.2021.09.049

Simonetta F. Highlights in CAR T-Cell Therapy from EHA2021: Paving the Way for Better Treatments. healthbook TIMES Onco Hema. 2021:(9):52-54.

6-months PFS rate was observed in patients receiving axi-cel while 17.1\% 6-months PFS was observed in those treated with tisa-cel.

\section{Does allogeneic HSCT still have a place next to CAR T-cell therapy?}

With the arrival of CAR T-cell therapy, the place for allogeneic hematopoietic stem cell transplantation (alloHSCT) in the treatment algorithm of DLBCL was increasingly questioned. Both treatment strategies have their advantages and inconveniences. ${ }^{10}$ Importantly, several important differences exist in the treatment algorithm for these two different strategies: in particular, while the response to salvage therapy is required for alloHSCT; it is not required for CAR T-cell therapy. The long-term overall survival (OS) is similar for both therapies, with $40 \%-50 \%$ for alloHSCT and 35\%-40\% for CAR T-cell therapy. Both strategies are associated with specific toxicities like graft versus host disease (GVHD) for alloHSCT ( 20\%) and cytokine release syndrome (CRS) and immune effector cell-associated neurotoxicity syndrome (ICANS) in CAR T-treated patients ( 5\%). While the cost is currently much higher for CAR T-cell therapy, this could partly be ascribed to the novelty and may change in the future. Overall, both treatments show a satisfying ratio of immune versus lymphoma effects and are available as standard of care (SOC) in most countries, although alloHSCT could be especially beneficial in patients with DLBCL in regions where CAR T-cell therapy is not available. Importantly, both treatments are not mutually exclusive and can be combined: allogeneic HSCT could be an option in case of relapse after CAR T-cell therapy or in patients who have not achieved complete remission $(<C R)$ or minimal residual disease negativity (MRD-), as well as in patients with poor bone marrow or with a therapy-related myeloid disorder. ${ }^{11}$ In addition, allogenic transplantation could be essential for R/R DLBCL patients when there is manufacturing failure of CAR T-cells or a CD19-negative relapse. ${ }^{11}$ 
Ciltacabtagene autoleucel (cilta-cel) is a second-generation BCMA-directed CAR T-cell therapy that has been investigated in the CARTITUDE-1 trial in RRMM patients $(n=97)$ who had received $\geq 3$ prior lines of therapy, with an ECOG $P S \leq 1$, and had received a $\mathrm{PI}$, an $\mathrm{IMiD}$, and anti-CD38 therapy prior to treatment with cilta-cel. ${ }^{20}$ The updated results of the CARTITUDE-1 trial presented by Dr Saad Z. Usmani showed that at a median followup of 18 months, ORR was $97.9 \%$ with a stringent complete response (sCR) rate of $80.4 \% .{ }^{21}$ Moreover, among MRDevaluable patients ( $n=61$ ), the great majority of patients (91.8\%) were MRD negative (threshold: $10^{-5}$ ). At a median follow-up of 18 months, the PFS was $66.0 \%$ and 18 -months OS was $80.9 \%$. Considering these very promising results, cilta-cel is currently under review for approval in the US and Europe..$^{22,23}$

There are currently ongoing trials evaluating cilta-cel in lenalidomide refractory patients with progressive disease after 1-3 lines of MM therapy (CARTITUDE-2) and comparing cilta-cel with standard treatment in relapsed and lenalidomiderefractory MM patients (CARTITUDE-4). ${ }^{24,25}$ Initial results from cohort A from the multicohort trial CARTITUDE-2 were presented at EHA2021 by Dr Mounzer E. Agha. ${ }^{26}$ A total of 20 patients in cohort A of the CARTITUDE-2 trial received a single cilta-cel infusion. The primary endpoint of the study was MRD negativity $\left(<10^{-5}\right.$ threshold). The secondary outcomes included ORR, duration of response (DOR), time and duration of MRD negativity and safety. At a median follow-up of 5.8 months, the ORR was $95 \%$, with $75 \%$ of patients achieving a complete response (CR) or better and $45 \%$ achieving a sCR. The median time to first response was 1 month and the median time to CR or better was 1.9 months. At data cutoff, the median DOR was not reached. All patients evaluable for MRD $(n=4)$ were MRD negative. Taken together, cilta-cel) led to early and deep responses in RRMM patients in this setting.

In conclusion, CAR T-cell therapy is, to use a metaphor employed by Prof. Cathrine Thieblemont at EHA2021, an additional stepping stone in the treatment of patients with DLBCL and RRMM. Hopefully, it can pave the path for patients with many other indications in the future, together with other available treatment approaches.
1. KYMRIAH ${ }^{\otimes}$ (tisagenleleucel). Product information FDA. [Accessed August 2021]. Available from: https:/ www.fda.gov/media/107296/download.

2. YESCARTA (axicabtagene ciloleucel). Product information. FDA. [Accessed August 2021]. Available from: https://www.fda.gov/media/108377/download. 3. BREYANZI ${ }^{\circledR}$ (lisocabtagene maraleucel). Product information. FDA. [Accessed August 2021]. Available from: https://www.fda.gov/media/145711/download. 4. KYMRIAH ${ }^{\circledast}$ (tisagenlecleucel). Product information. Swissmedic. [Accessed August 2021] Available from: Swissmedic. [Accessed August
https://www.swissmedicinfo.ch.

5. YESCARTA (axicabtagene ciloleucel). Product in formation. Swissmedic. [Accessed August 2021]. Available from: https://www.swissmedicinfo.ch.

6. Schuster SJ, Bishop MR, Tam CS, et al. Tisagenlecleucel in Adult Relapsed or Refractory Diffuse Large B-Cell Lymphoma. N Engl J Med. 2019;380(1):45-56. doi:10.1056/NEJMoa1804980

7. Locke FL, Ghobadi A, Jacobson CA, et al. Long 7. Locke FL, Ghobadi A, Jacobson CA, et al. Long
term safety and activity of axicabtagene ciloleu term safety and activity of axicabtagene ciloleu-
cel in refractory large B-cell lymphoma (ZUMAcel in refractory large B-cell lymphoma (ZUMA
1): a single-arm, multicentre, phase 1-2 trial. Lancet Oncol. 2019;20(1):31-42. doi:10.1016/S1470 2045(18)30864-7

8. Abramson JS, Palomba ML, Gordon LI, et al. Lisocabtagene maraleucel for patients with relapsed or refractory large B-cell Iymphomas (TRANSCEND NHL 001): a multicentre seamless design study. Lancet. 2020;396(10254):839-852. doi:10.1016/ Lancet. 2020;396(10254)

S0140-6736(20)31366-0
9. Le Gouill S, Bachy E, Di Blasi R, et al. First Results 9. Le Gouill S, Bachy E, Di Blasi R, et al. First Results
of Patients Treated with CAR-T Cells and Enrolled in DESCAR-T Registry, a French Real-life Database for CAR-T Cells in Hematologic Malignancies. Presented at the: EHA2O21 Virtual Congress; 9-17 June 2021 Oral presentation S216.

10. Shah NN, Hamadani M. Is There Still a Role for Allogeneic Transplantation in the Management of Lymphoma?. J Clin Oncol. 2021;39(5):487-498. doi:10.1200/JCO.20.01447

11. Dreger P, Fenske TS, Montoto S, Pasquini MC, 11. Dreger P, Fenske TS, Montoto S, Pasquini MC
Sureda A, Hamadani M. Cellular Immunotherapy for Sureda A, Hamadani M. Cellular Immunotherapy for
Refractory Diffuse Large B Cell Lymphoma in the Chimeric Antigen Receptor-Engineered T Cell Era: Still Role for Allogeneic Transplantation?. Biol Blood Mar- row Transplant. 2020;26(4):e77-e85. doi:10.1016/j. bbmt.2019.12.771

12. Jacobson C, Chavez JC, Sehgal AR, et al. Primary Analysis of Zuma-5: A Phase 2 Study of Axicabtagene Ciloleucel (Axi-Cel) in Patients with Relapsed/Refrac tory (R/R) Indolent Non-Hodgkin Lymphoma (iNHL). Blood. 2020:136(Suppl_1):40-41. doi:10.1182 blood-2020-136834

13. Ghione P, Anik R Patel, Sabela Bobillo, et al. A com13. Ghione P, Anik R Patel, Sabela Bobillo, et al. A comparison of clinical outcomes from ZUMA-5 (axicabta-
gene ciloleucel) and the international SCHOLAR-5 gene ciloleucel) and the international SCHOLAR-5
external control cohort in relapsed/refractory follicular lymphoma (R/R FL). Presented at the: EHA2O21 Virtual Congress; 9-17 June 2021. Oral presentation LB1904.

14. $A B C E M A^{\circledR}$ (idecabtagene vicleucel). Product information. FDA. [Accessed August 2021]. Available from: https://www.fda.gov/media/147055/download.

15. Munshi NC, Anderson LD, Shah N, et al. Idecab15. Munshi NC, Anderson LD, Shah N, et al. Idecabtagene Vicleucel in Relapsed and Refractory Mul-
tiple Myeloma. N Engl J Med. 2021;384(8):705-716. tiple Myeloma. N Engl J Med. 202

doi:10.1056/NEJMoa2024850
16. Oriol A, San Miguel J, Kansagra A, et al. Idecabtagene Vicleucel (Ide-cel, BB2121), a BCMA-directed CAR T Cell Therapy, in Patients with Relapsed and Refractory Multiple Myeloma: Updated KarMMa Results. Presented at the: EHA2021 Virtual Congress; 9-17 June 2021. Poster presentation EP1009.

17. Efficacy and Safety Study of bb2121 Versus Standard Regimens in Subjects With Relapsed sus Standard Regimens in Subjects With Relapsed and Refractory Multiple Myeloma (RRMM) (KarM-
Ma-3). ClinicalTrials.gov. [Accessed August 2021]. Ma-3). ClinicalTrials.gov. [Accessed August 2021].
Available from: https://clinicaltrials.gov/ct2/show/ NCT03651128? cond=KarMMa\&draw $=2 \&$ rank $=3$. 18. A Study to Evaluate the Safety of bb2121 in Subjects With High Risk, Newly Diagnosed Multip le Myeloma (NDMM) (KarMMa-4). ClinicalTrials.org. [Accessed August 2021]. Available from: https://clinicaltrials. gov/ct2/show/NCT04196491?cond=KarM Ma\&draw $=2 \&$ rank=1

19. Safety and Efficacy of bb2121 (Ide-cel) Combinations in Multiple Myeloma (KarMCombinations in Multiple Myeloma (KarM-
Ma-7). [Accessed August 2021]. AvailaMa-7). [Accessed August 2021]. Availa-
ble from: https://Clinicaltrials.gov/ct2/show/ NCT04855136? cond=KarMMa\&draw $=2$ \&rank $=2$ 20. Berdeja JG, Madduri D, Usmani SZ, et al. Cilta- cabtagene autoleucel, a B-cell maturation antigendirected chimeric antigen receptor T-cell therapy in patients with relapsed or refractory multiple myeloma (CARTITUDE-1): a phase 1b/2 open-label study. Lancet. doi:10.1016/S0140-6736(21)00933-8

21. Usmani S, Berdeja J, Madduri D, et al. Updated CARTITUDE-1 Results of Ciltacabtagene Autoleucel, a B-cell Maturation Antigen-directed Chimeric AntiBen Receptor T-cell Therapy, in Relapsed/Refractory gen Receptor T-cell Therapy, in Relapsed/Refractory Multiple Myelome. Presented at the: EHA2021 Virtual Congress; 9-17 June 2021. Poster presentation EP964

22. Cilta-Cel Earns FDA Priority Review for Relapsed/ Refractory Multiple Myeloma. Cancer network. [Accessed August 2021]. Available from: https://www. cancernetwork.com/view/cilta-cel-earns-fda-priorityreview-for-relapsed-refractory-multiple-myeloma. 23. Accelerated Assessment of Cilta-Cel Announced by the EMA. MultipleMyelomaHub. [Accessed August 20211. Avalable from: htps//multiplemyelomast 2021]. Av. Multiple Myeloma Hib. [Accessed Ausust ed-assessment-ofcilta-cel-announced-by-the-ema.

24. A Study of JNJ-68284528, a Chimeric Antigen Receptor T Cell (CAR-T) Therapy Directed Against Bcell Maturation Antigen (BCMA) in Participants With Multiple Myeloma (CARTITUDE-2). ClinicalTrials.org. [Accessed August 2021]. Available from: https://clinicaltrials.gov/ct2/show $/$ NCT04133636?term $=$ CARTITUDE\&draw $=2 \&$ rank $=3$.

25. A Study Comparing JNJ-68284528, a CAR-T Therapy Directed Against B-cell Maturation Antigen (BCMA), Versus Pomalidomide, Bortezomib and Dexamethasone (PVd) or Daratumumab, Pomalidomide and methasone (PVd) or Daratumumab, Pomalidomide and
Dexamethasone (DPd) in Participants With Relapsed and Lenalidomide-Refractory Multiple Myeloma (CARTITUDE-4). ClinicalTrials.org. [Accessed August 2021]. Available from: https://clinicaltrials.gov/ct2/show/ NCT04181827?term=CARTITUDE\&draw $=2 \&$ rank $=2$. 26. Agha ME, Cohen A, Madduri D, et al. Efficacy and Safety of the BCMA-directed CAR-T Cell Therapy, CilSaln tacabtagene Autoleucel, in Patients with Progressive Multiple Myeloma After 1-3 Prior Lines of Therapy: EHA2021 Virtual Congress; 9-17 June 2021. Oral presentation S190. 